\title{
Sondages et étude des ruines de la chapelle préromane sur le site de San Peyre (Hérault)
}

\section{Élisabeth Astruc}

\section{(2) OpenEdition \\ 1 Journals}

Édition électronique

URL : https://journals.openedition.org/cem/17818

DOI : $10.4000 /$ cem. 17818

ISSN : 1954-3093

\section{Éditeur}

Centre d'études médiévales Saint-Germain d'Auxerre

\section{Référence électronique}

Élisabeth Astruc, «Sondages et étude des ruines de la chapelle préromane sur le site de San Peyre (Hérault) », Bulletin du centre d'études médiévales d'Auxerre | BUCEMA [En ligne], 24.2 | 2020, mis en ligne le 19 décembre 2020, consulté le 22 septembre 2022. URL : http://journals.openedition.org/cem/ 17818 ; DOI : https://doi.org/10.4000/cem.17818

\section{Ce document a été généré automatiquement le 22 septembre 2022.}

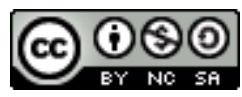

Creative Commons - Attribution - Pas d'Utilisation Commerciale - Partage dans les Mêmes Conditions 4.0 International - CC BY-NC-SA 4.0

https://creativecommons.org/licenses/by-nc-sa/4.0/ 


\section{Sondages et étude des ruines de la chapelle préromane sur le site de San Peyre (Hérault)}

Élisabeth Astruc

San Peyre se situe sur la commune de Saint-Maurice-Navacelles, au nord du département de l'Hérault, en bordure méridionale du plateau du Larzac (fig. 1).

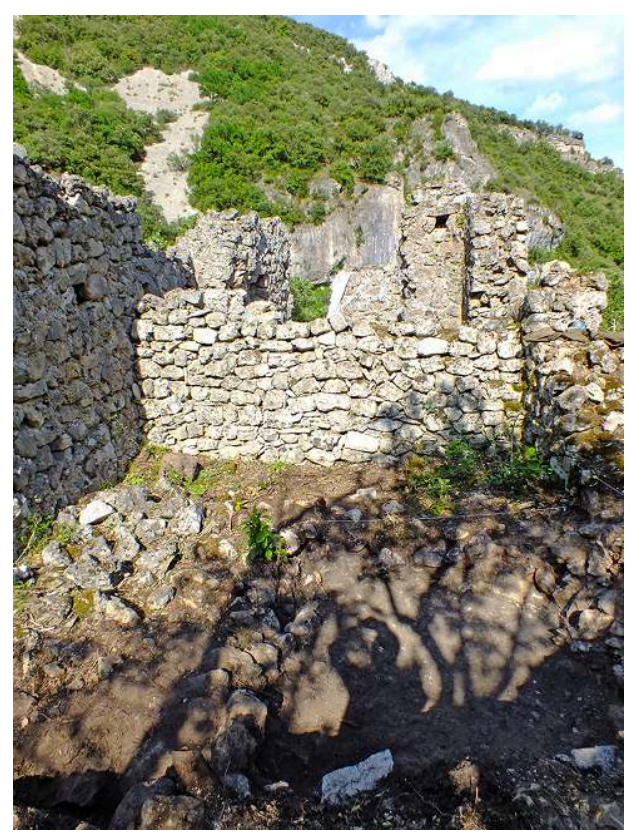


Fig. 1 - Localisation du site sur fond de carte IGN au 1/25000.

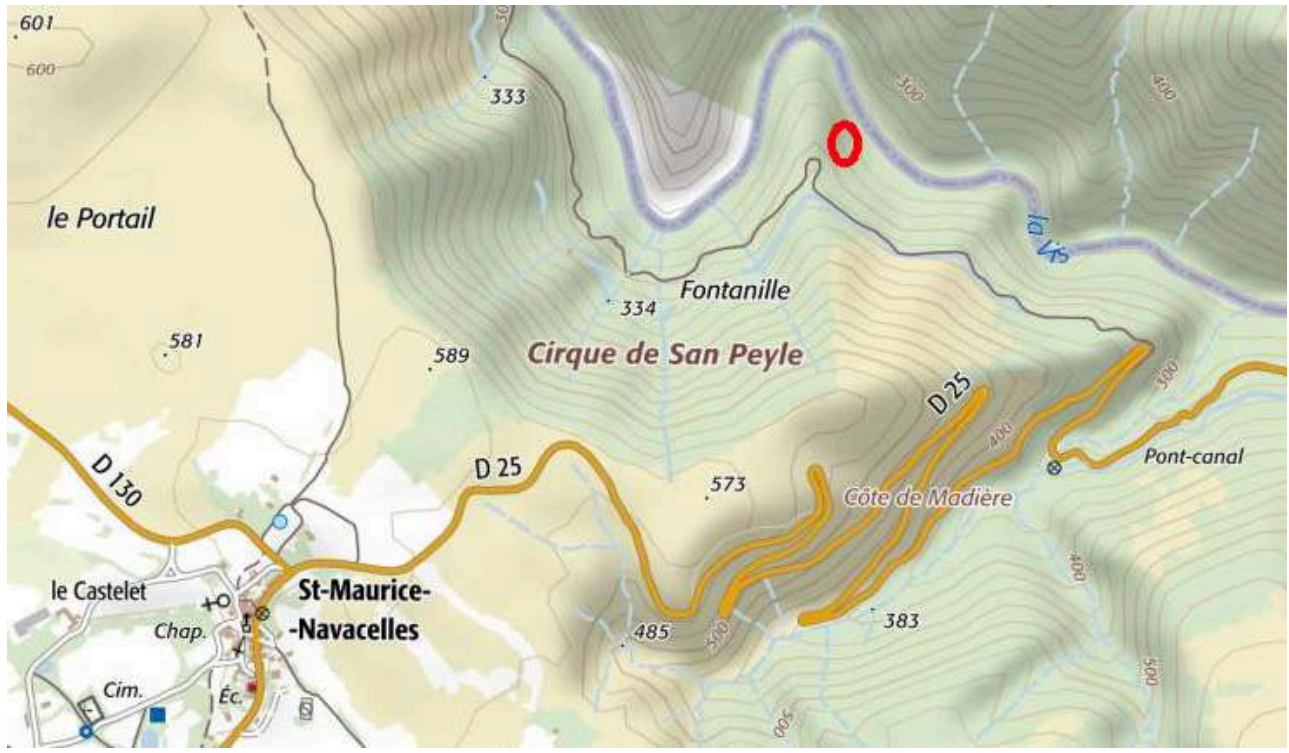

$C^{\prime}$ est au nord-est de cette commune, en suivant la départementale $\mathrm{n}^{\circ} 25$ en direction de Madières, que le site est perché sur un piton surplombant les gorges de la Vis (fig. 2).

Fig. 2 - Vue depuis le promontoire sur la route D25 (cl. É. Astruc).

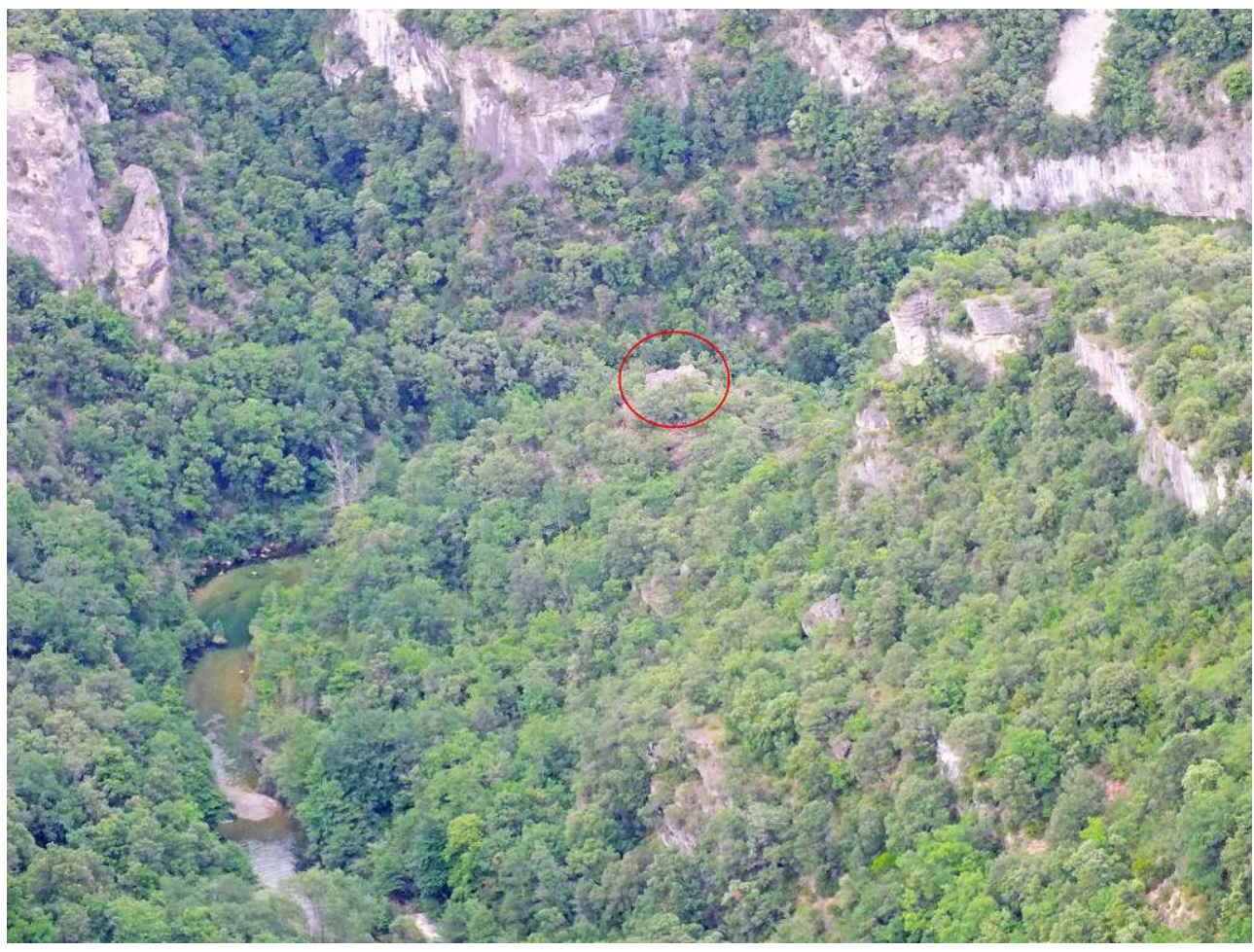

3 L'édifice religieux est localisé sur une partie du territoire correspondant aux anciennes limites du diocèse de Lodève.

4 L'étude du site s'inscrit dans une recherche globale mise en place sur le plateau du Larzac par Agnès Bergeret (Inrap) ${ }^{1}$, visant à comprendre l'organisation de ce territoire au haut Moyen Âge, mais également dans le cadre d'une thèse de doctorat en histoire 
de l'art médiéval ${ }^{2}$. L'intervention archéologique s'est déroulée en juin $2014^{3}$; elle s'est concentrée sur l'église et sur la petite terrasse attenante (fig. 3).

Fig. 3 - Localisation des zones de sondages (DAO É. Astruc, 2014).

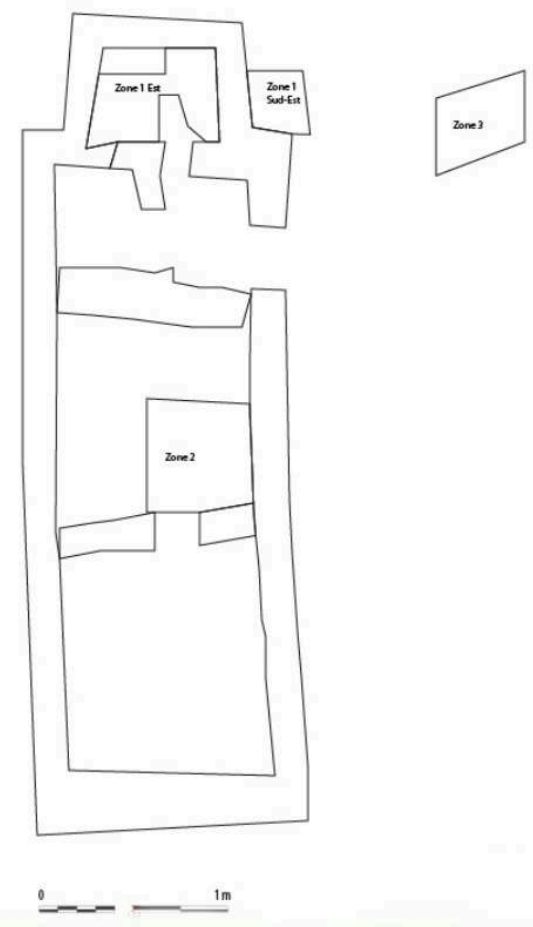

5 L'objectif principal était de dégager le plan de l'édifice, qui présente des caractéristiques propres aux édifices ruraux bâtis avant l'an Mil, dits préromans, et d'en comprendre les espaces de circulation. Les structures apparentes proches de la chapelle n'ont pas été étudiées et semblent correspondre à un habitat. Elles seront analysées lors d'une prochaine campagne.

En ce qui concerne la documentation, peu d'informations sont données sur l'édifice religieux. L'abbé Alzieu a dépouillé l'ensemble des textes se rapportant aux anciens diocèses que comptait le département de l'Hérault ${ }^{4}$. Son ouvrage sur les églises de l'ancien diocèse de Lodève publié en 1998 recense de nombreux monuments sur la commune de Saint-Maurice-Navacelles, dont Notre-Dame de la Peyre ${ }^{5}$. Cette dernière est mentionnée en 1410 dans le testament d'Arnaud Soubeiras, qui lègue deux livres de cire à quatre églises de Madières. L'abbé Guichard, qui rapporte la mention dans les Madonnes, pense qu'il s'agit de la vieille église du château de Madières. Cependant, deux églises sont visibles dans la localité, la plus ancienne se situant dans le cimetière, l'autre ayant été reconstruite $\mathrm{au} \mathrm{XIX}{ }^{\mathrm{e}}$ siècle. La mention du début $\mathrm{du} \mathrm{XV}^{\mathrm{e}}$ siècle pourrait donc correspondre au site étudié avec une toponymie identique. Ce seul document nous renseigne sur la titulature de l'église, dédiée à la Vierge.

7 Une historienne locale, Georgette Milhau, a publié en 1998 un ouvrage intitulé Navacelles, dans lequel elle indique la présence de ruines d'une église au « lo roc de San Peyle », où un bénitier avait été creusé dans le roc $^{6}$. Elle l'attribue à des ermites venus se "réfugier dans ces lieux sauvages". Le statut de l'édifice a été affiné grâce aux recherches archéologiques. L'absence de sépulture aux abords des vestiges indique, en 
effet, qu'il s'agit probablement d'une chapelle annexe dépendant de l'église paroissiale de Saint-Maurice-Navacelles.

8 Les sondages ont mis en évidence trois phases d'occupation (fig. 4).

Fig. 4 - Plan de la chapelle. a. premier état des $\mathrm{X}^{\mathrm{e}}-\mathrm{Xl} \mathrm{e}^{\mathrm{e}}$ siècles; $\mathrm{b}$. deuxième état des $\mathrm{Xl}^{\mathrm{e}}-\mathrm{XIII}{ }^{\mathrm{e}}$ siècles ; c. troisième état des $X V I^{e}-X_{1}{ }^{e}$ siècles (DAO É. Astruc, 2014).
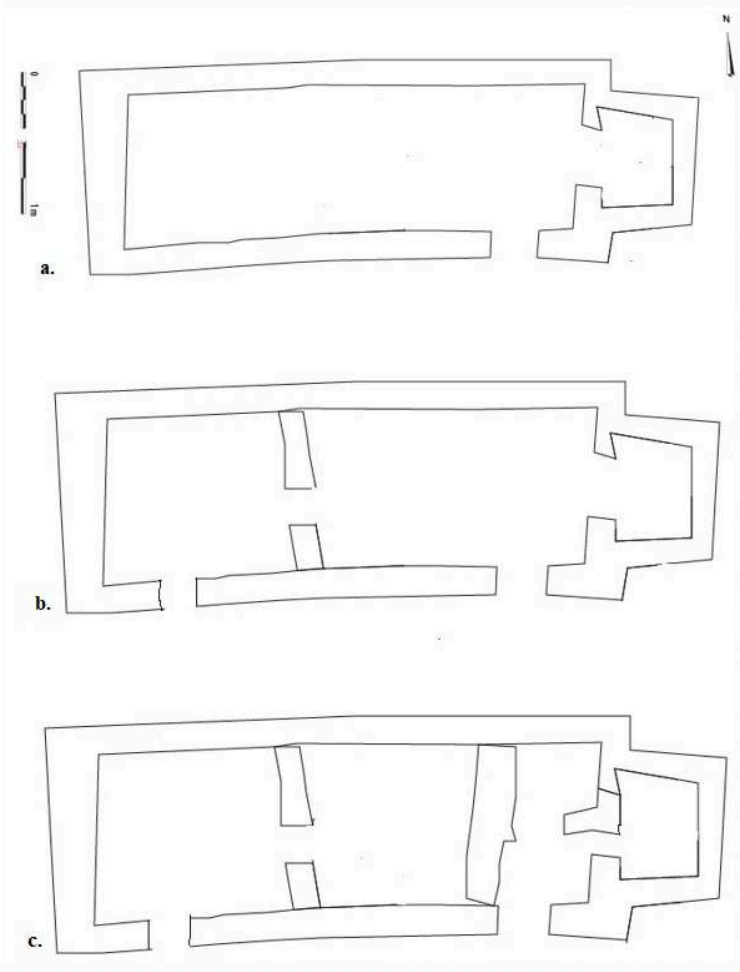

\section{Un premier édifice des $\mathrm{X}^{\mathrm{e}}-\mathrm{XI}^{\mathrm{e}}$ siècles}

9 L'emplacement choisi pour ériger la chapelle est le plus élevé de la terrasse. Il surplombe la rivière de la Vis. Le substrat rocheux ne correspond pas à une surface plane mais comporte plusieurs creux. Une dalle en mortier très compact avec des inclusions de silex a été coulée afin d'obtenir un sol plus régulier.

10 L'édifice est d'assez vastes proportions, composé d'une nef à vaisseau unique ouvrant sur un chevet quadrangulaire orienté (fig. 5). 
Fig. 5 - Vue intérieure vers l'est (cl. É. Astruc, 2014).

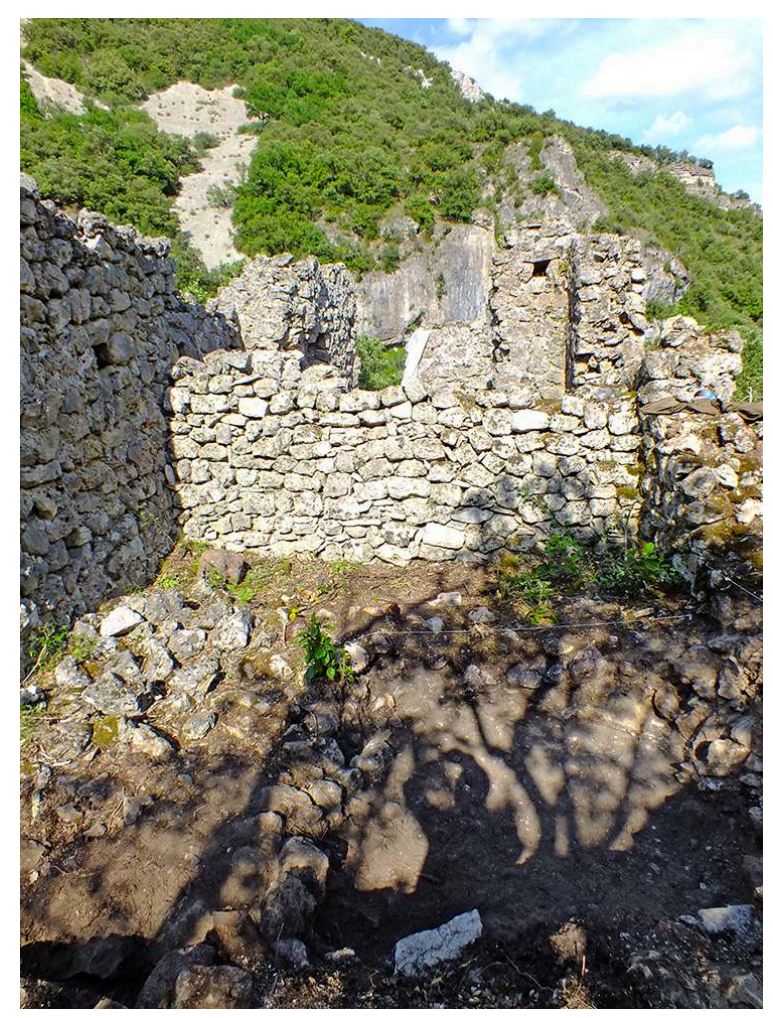

11 Les dimensions hors-œuvre s'échelonnent en longueur entre 15,40 m et 16,60 m pour une largeur à l'ouest de 5,30 m et à l'est de 3,35 m.

12 La nef mesure 13,30 m de longueur hors-œuvre pour le mur sud et 13,70 m pour le pan nord. Cette différence est sûrement due à l'arbre qui a poussé dans l'angle intérieur formé par les murs ouest et nord. La largeur est de 5,30 m à l'ouest et de 5,20 $\mathrm{m}$ à l'est. Les montants de l'arc triomphal font une forte avancée à la corde, de $1 \mathrm{~m}$ et 1,20 $\mathrm{m}$ sur leurs faces ouest et de 0,60 m sur leurs faces est. L'écart entre ces deux maçonneries est de 1,50 m seulement ; il correspond à l'entrée du chœur.

13 Ce dernier est de plan trapézoïdal, très évident à l'intérieur avec $2 \mathrm{~m}$ de longueur dans l'œuvre pour le mur nord et 1,90 m au sud, pour une largeur de 2,60 m à l'ouest et $2,20 \mathrm{~m}$ à l'est. C'est le seul édifice du territoire du diocèse de Lodève à présenter une forme aussi marquée.

14 La limite entre nef et chevet est signifiée à l'extérieur par un décrochement des murs gouttereaux de 0,80 m (fig. 6). 
Fig. 6 - Décrochement du mur gouttereau nord (cl. É. Astruc, 2014).

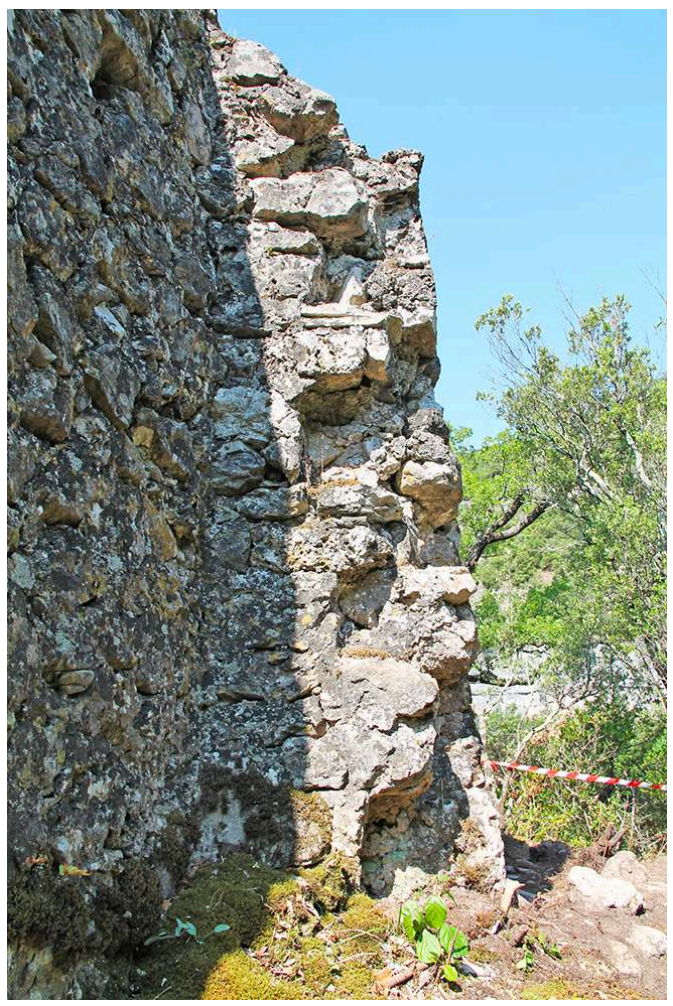

15 L'épaisseur des murs s'échelonne entre 0,60 et 1,20 m. L'accès s'effectuait par une porte percée dans la façade méridionale à $1,90 \mathrm{~m}$ du décrochement sud. L'ouverture mesure $1,20 \mathrm{~m}$ de large, dans un gouttereau sud épais de 0,65 $\mathrm{m}$. Les montants conservent l'emplacement pour la barre en bois qui verrouillait la porte. Ce creusement visible dans le mur méridional a été identifié par Georgette Milhau comme étant un bénitier. Aucune fenêtre n'a été conservée. Pourtant, la nef devait au moins être éclairée par une baie dans le mur sud, car un linteau monolithe en tuf creusé en arc plein cintre a été découvert dans les remblais de la nef (fig. 7). 
Fig. 7 - Linteau (cl. É. Astruc, 2014).

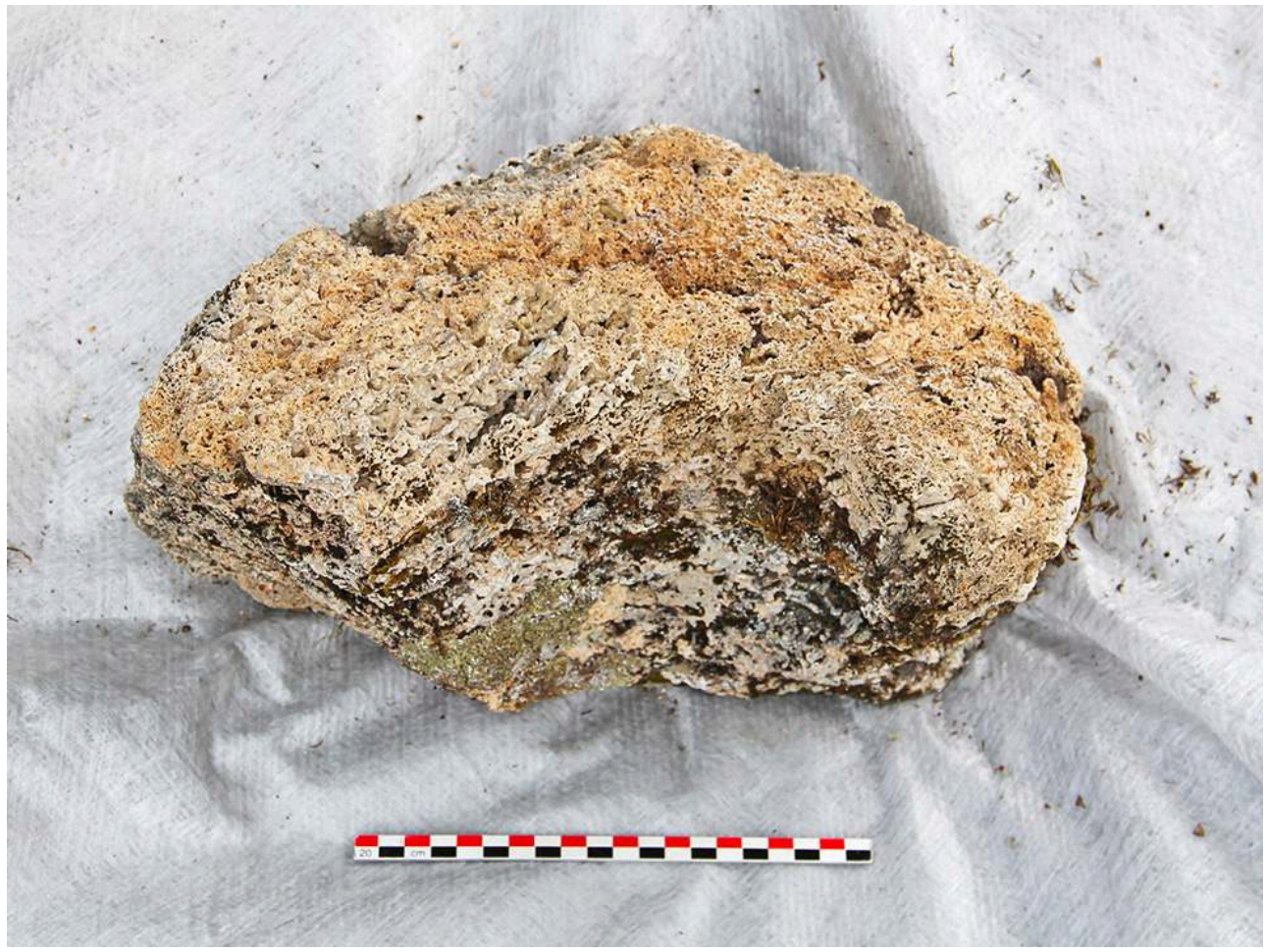

16 Il présente des caractéristiques identiques à ceux toujours en place dans les édifices de la région construits aux $\mathrm{x}^{\mathrm{e}}-\mathrm{XI}^{\mathrm{e}}$ siècles ${ }^{7}$.

17 Les murs sont bâtis en moellons disparates de calcaire local avec inclusion de blocs de tuf, grossièrement équarris ou employés tels quels, disposés en assises irrégulières. Un mortier de chaux compact est utilisé comme liant. Il a été lissé sur les moellons et devait faire office d'enduit. La façade septentrionale présente une assise disposée en opus spicatum de 1,41 m de long (fig. 8). 
Fig. 8 - Assise disposée en opus spicatum dans les maçonneries du mur septentrional de la nef (cl. É. Astruc, 2014).

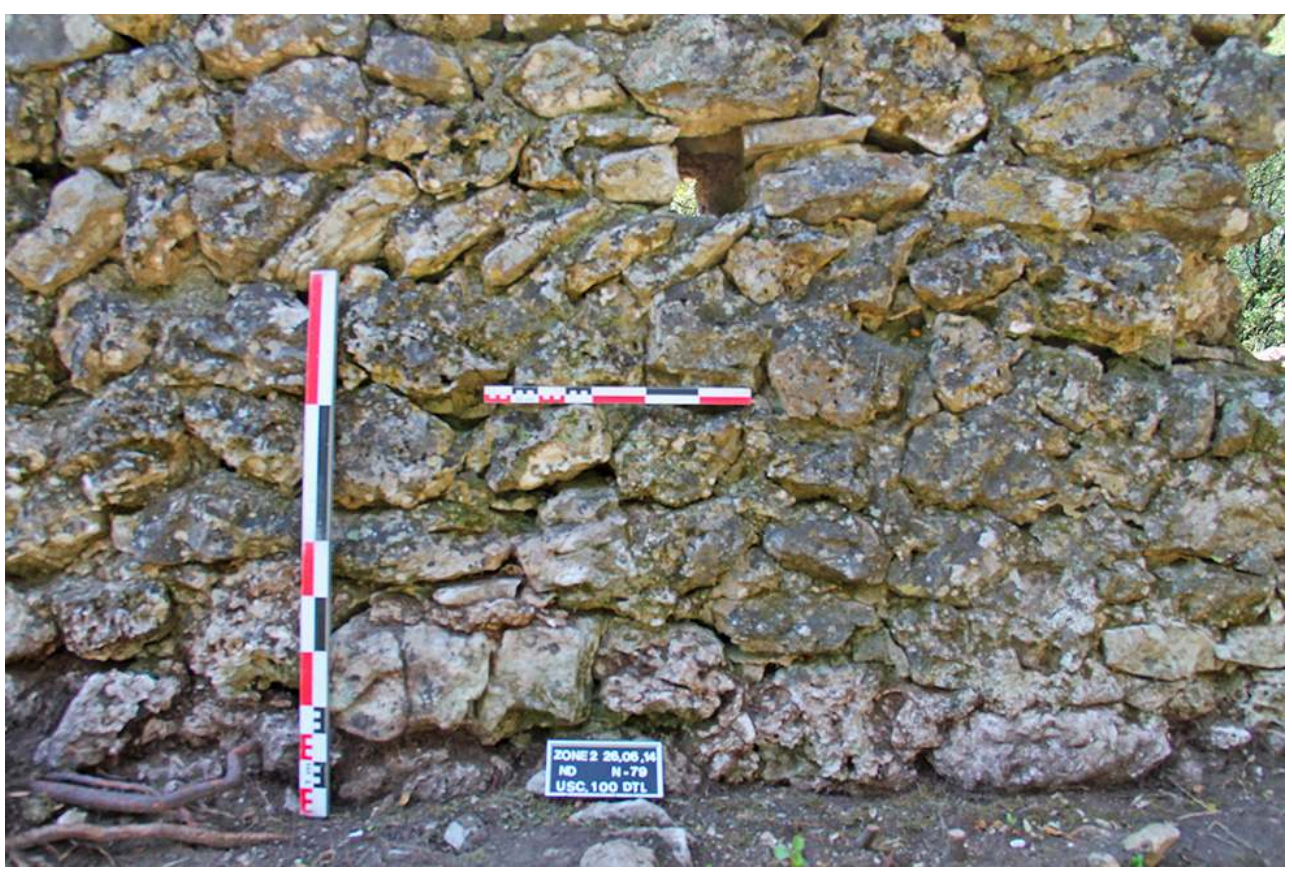

Des pierres de calage ont été mises en place entre les rangées d'assises afin de renforcer la construction. Néanmoins, cela semble avoir eu l'effet inverse: ces dernières ont éclaté à cause du gel, ce qui a fragilisé l'édifice provoquant l'écroulement des six chaînes d'angle. Ce système de construction ne s'observe que pour cet édifice dans le diocèse de Lodève. Des trous de boulins traversants, pour certains non bouchés, sont toujours visibles sur les murs nord, est et sud. Un soin particulier a été apporté aux éléments les plus importants de l'édifice : les chaînes d'angle ainsi que l'arc triomphal sont montés en blocs de tuf bien taillés.

19 Cet édifice de taille imposante pour une chapelle présente toutes les caractéristiques architecturales propres aux constructions des $\mathrm{x}^{\mathrm{e}}-\mathrm{XI}^{\mathrm{e}}$ siècles en Languedoc méditerranéen. Les vastes dimensions de sa nef laissent supposer que les fidèles y étaient nombreux. L'église desservait probablement l'habitat qui la jouxte ainsi que les hameaux disséminés sur le territoire entre Saint-Maurice-Navacelles et Madières.

\section{Des aménagements aux $\mathrm{XII}{ }^{\mathrm{e}}-\mathrm{XIII}{ }^{\mathrm{e}}$ siècles}

À partir des $\mathrm{XII}^{\mathrm{e}}$-XIII ${ }^{\mathrm{e}}$ siècles, le plan de l'église est modifié. Sa nef est divisée en deux par un mur de refend orienté nord-sud, positionné à $4,70 \mathrm{~m}$ du parement est de la façade occidentale. Épais de $0,70 \mathrm{~m}$, le mur est fait de pierres bien taillées, disposées en assises régulières, et liées à l'aide d'un mortier plus fin et moins compact que celui employé dans les autres maçonneries. Une porte large de $0,85 \mathrm{~m}$ y a été percée. Les montants se composent de gros parpaings de tuf bien taillés. Les premiers blocs présentent des creusements sur leur face sud et nord qui devaient accueillir les charnières de la porte (fig. 9). 
Fig. 9 - Porte bouchée dans le mur de refend orienté nord-sud (cl. É. Astruc, 2014).

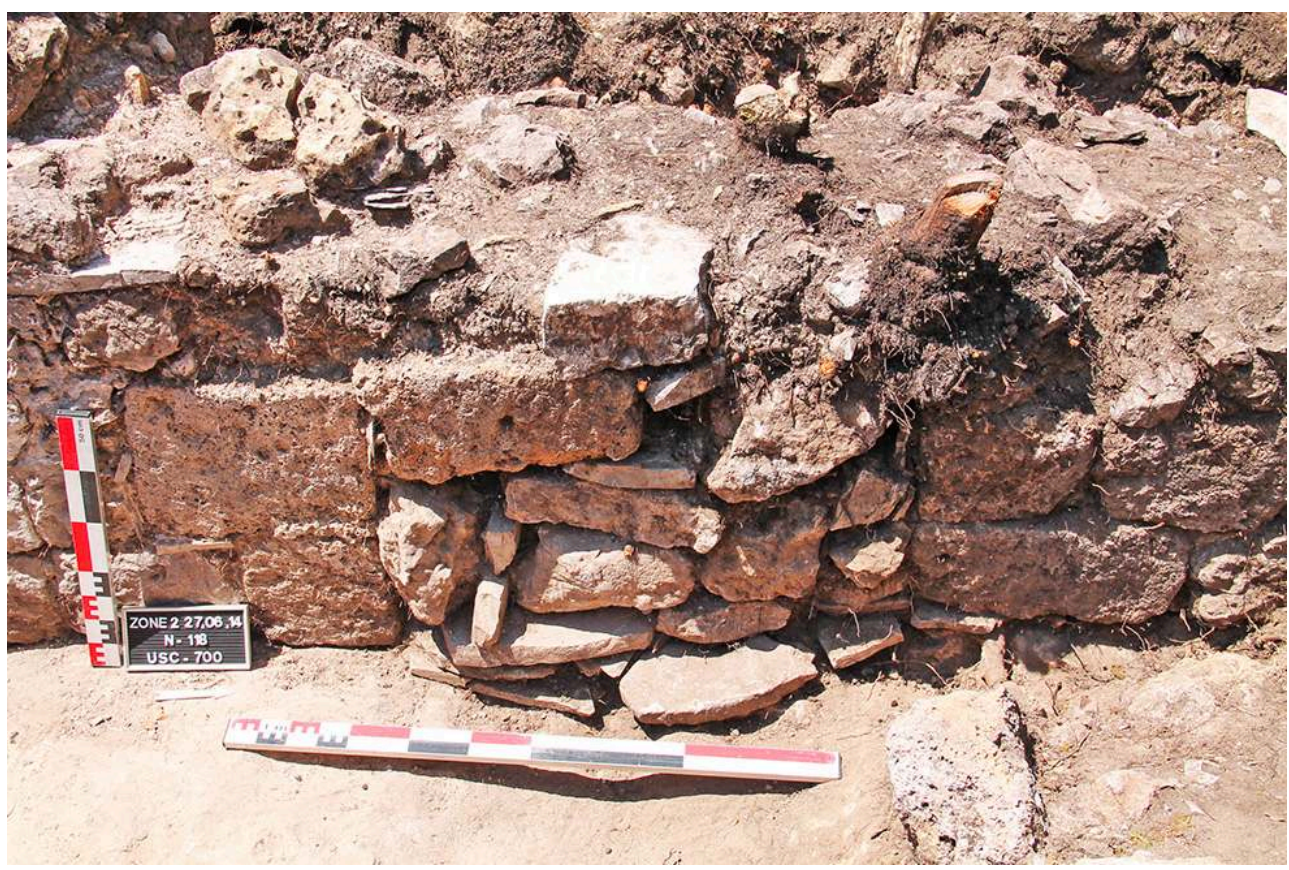

Plusieurs tessons de céramique ont été découverts en dégageant la porte. Ils sont datés entre le $\mathrm{x}^{\mathrm{e}}$ et le $\mathrm{XII}^{\mathrm{e}}$ siècle $^{8}$. La porte était bouchée à l'aide des gros blocs de tuf appartenant aux montants et de pierres de calage. Des tessons, datés probablement du $\mathrm{XI}^{\mathrm{e}}$ siècle, ont été mis au jour dans ce bouchage.

Les $\mathrm{XII}^{\mathrm{e}}$-XIII ${ }^{\mathrm{e}}$ siècles voient un rétrécissement de la nef, ce qui semble indiquer une baisse de la démographie. Agnès Bergeret a constaté une réduction similaire en 2004-2005 lors de sondages archéologiques à Saint-Clément-de-Mans, sur la commune de Soubès.

La nouvelle pièce ménagée à l'ouest pourrait correspondre au logement du desservant. Un bâtiment prend alors appui au sud-ouest de la chapelle. Une porte ouverte dans la façade sud-ouest en permettait l'accès. Ce bâtiment, qui n'a pas fait l'objet d'étude pour le moment, semble n'offrir qu'une unique entrée depuis l'intérieur de la chapelle.

\section{Nouvelles transformations à la période moderne}

L'étude du chœur a révélé un agencement de dalles visibles quasiment sur l'ensemble du sol. Ces pierres taillées après extraction se sont avérées être des fragments de la table d'autel (fig. 10). 
Fig. 10 - Fragments de la table d'autel (cl. É. Astruc, 2014).

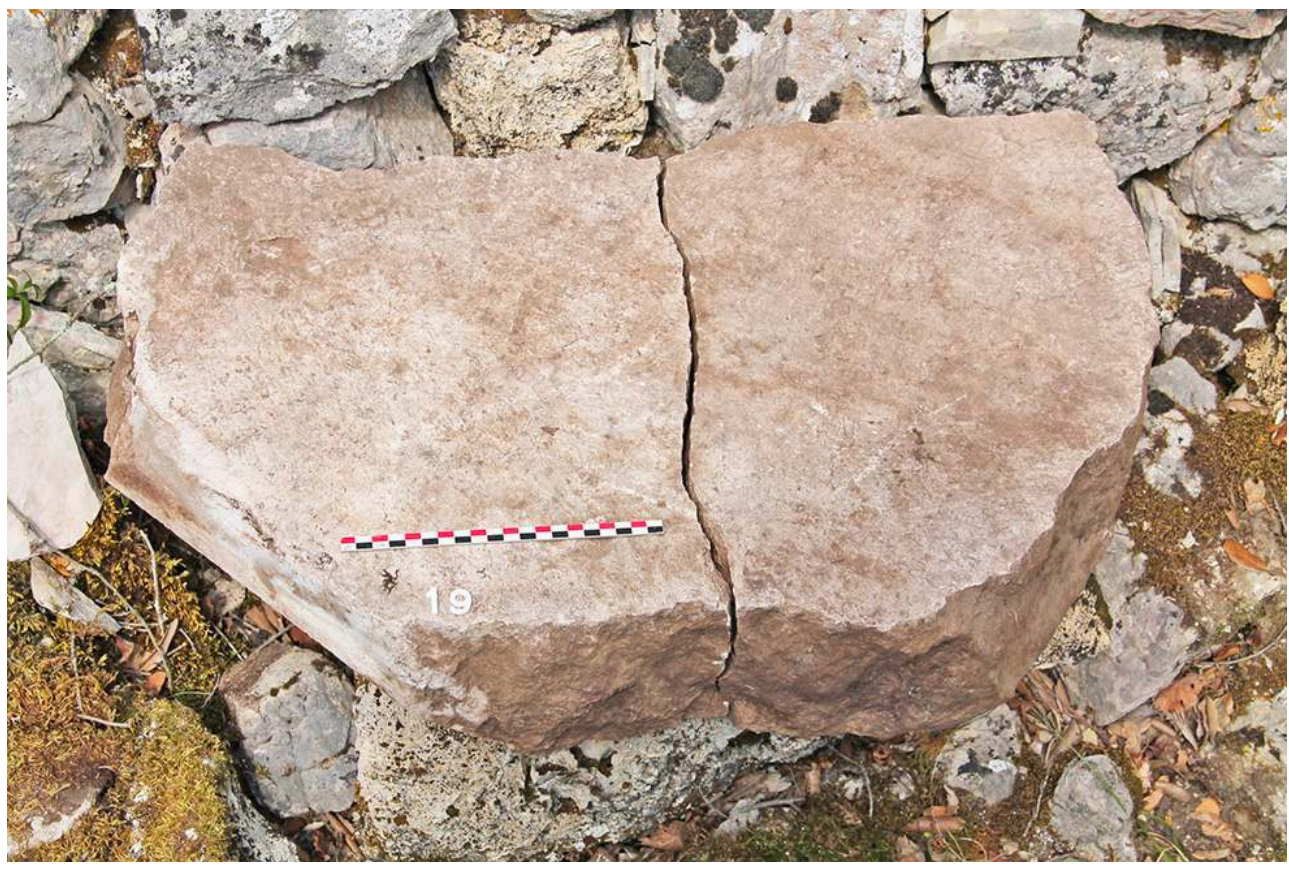

La mensa monolithe, de $18 \mathrm{~cm}$ d'épaisseur, est d'aspect fruste, en calcaire gris local. Les angles ont été biseautés (fig. 11).

Fig. 11 - Détail d'un angle de la table d'autel (cl. É. Astruc, 2014).

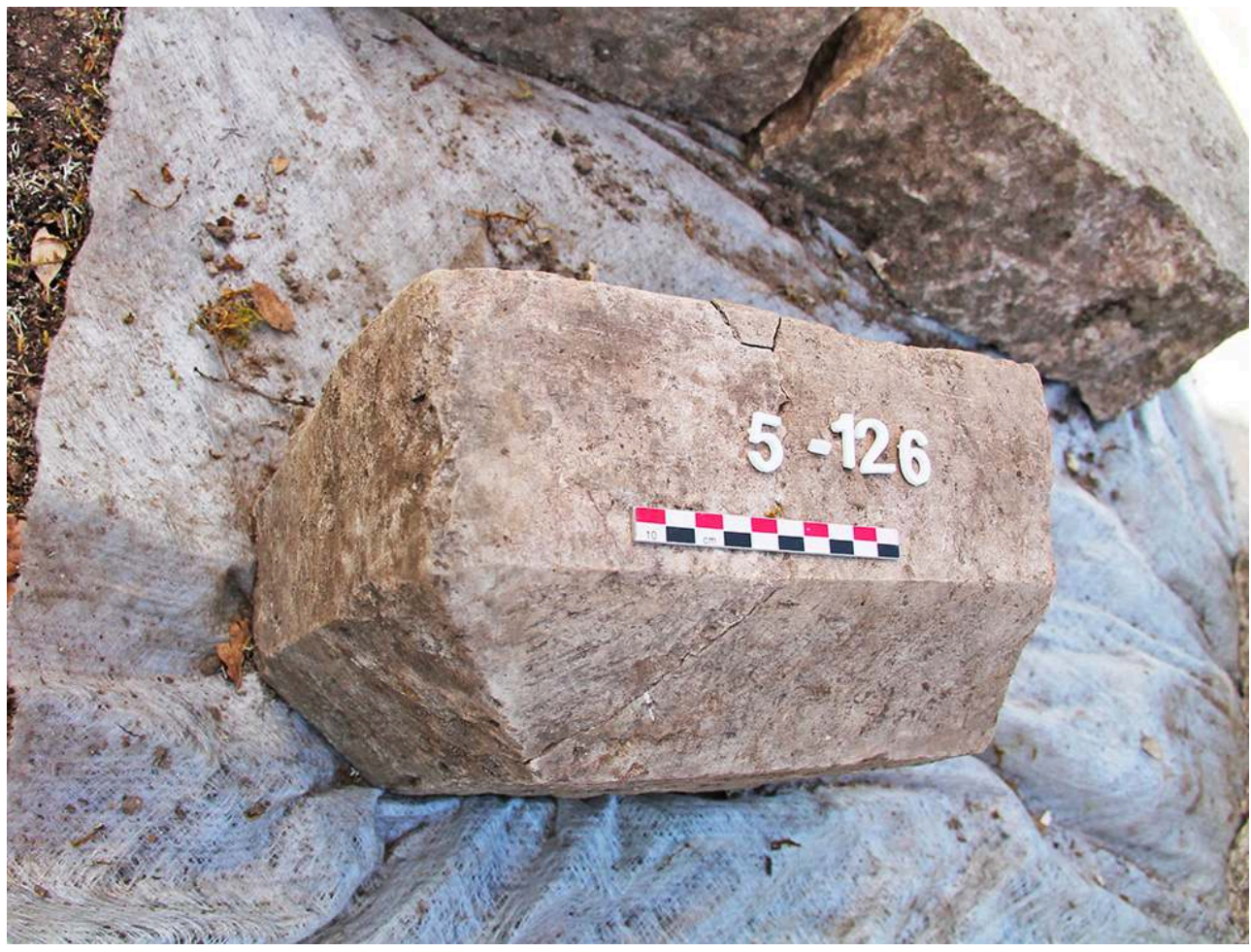

Elle a été brisée et ses morceaux (cinq ont été découverts) ont été utilisés comme dalles de sol. Le support de la table, probablement maçonné, n'a pas été retrouvé. Il s'agit vraisemblablement de la table d'autel primitive de la chapelle. Le sol a aussi livré une 
jatte quasi complète (datée des XVIII ${ }^{\mathrm{e}}$-XIX ${ }^{\mathrm{e}}$ siècles), ainsi qu'une monnaie : un liard à l'H frappé à la fin du XvI siècle, très répandu en France durant les guerres de religion? ${ }^{\mathrm{e}}$.

Le bâtiment subit des modifications qui laissent penser que l'édifice ne possède plus de fonction religieuse, mais aurait été transformé en habitat. Un mur massif, orienté nordsud, composé de moellons et de blocs de tuf de remploi, est édifié à 2,20 m de l'entrée du chœur. Un léger décrochement dans la maçonnerie répond à l'ajout d'un pan de mur qui vient fermer de manière plus marquée l'entrée du chœur $(0,60 \mathrm{~m})$. Cet agencement met en avant la création d'une petite pièce. En dégageant le mur, des tessons d'un même toupin, datés du XIX ${ }^{e}$ siècle, ont été découverts.

À partir de la période moderne, on peut donc supposer que la chapelle perd sa fonction religieuse, avec la destruction de la table d'autel remployée dans le sol, pour se transformer peu à peu en habitat, avec une occupation qui s'étend jusqu'au XIX ${ }^{\mathrm{e}}$ siècle.

\section{Synthèse et attentes}

La particularité et l'intérêt du site de San Peyre sont liés aux bâtiments annexes qui jouxtent l'édifice et qui correspondent à un habitat, ainsi qu'à la conservation d'une bonne partie de son élévation. L'étude architecturale et stratigraphique indique une occupation du site des $\mathrm{x}^{\mathrm{e}}-\mathrm{XI}^{\mathrm{e}}$ siècles jusqu'au $\mathrm{XIX}^{\mathrm{e}}$ siècle. Si le texte du début du $\mathrm{xv}^{\mathrm{e}}$ siècle, cité plus haut, concerne effectivement la chapelle, celle-ci était alors toujours en activité. C'est à partir des $\mathrm{XVII}^{\mathrm{e}}-\mathrm{XVIII}{ }^{\mathrm{e}}$ siècles qu'elle connaît des modifications en vue d'une transformation en habitat. Les découvertes à venir devraient permettre de mieux cerner les évolutions architecturales de l'édifice, mais surtout de comprendre son interaction avec les différents bâtiments attenants qui affleurent le sol actuel.

La future campagne de fouilles, qui devrait se dérouler en juillet 2021, permettra d'affiner la compréhension des espaces de circulation à l'intérieur de la chapelle grâce à l'étude du bâtiment la jouxtant au sud. Le cœur de la campagne se concentrera sur l'examen des vestiges annexes disposés sur la terrasse, afin, notamment, de déterminer leurs fonctions et si leur implantation s'est faite concomitamment à la construction de l'église ou de l'une de ses phases de modification.

Reçu : 31 août 2020 - Accepté : 24 novembre 2020

\section{NOTES}

1. Agnès Bergeret a travaillé sur trois sites présentant un édifice religieux accompagné d'un habitat: Saint-Vincent, situé à Saint-Maurice-Navacelles; Saint-Clément-de-Mans, localisé à Soubès; et Saint-Martin, sis à La Vacquerie-et-Saint-Martin-de-Castries. Voir, notamment, A. BERGERET, "Saint-Maurice-Navacelles (Hérault). Saint-Vincent de Soulages", Archéologie médiévale, 39 (2009), p. 235 ; 40 (2010), p. $315 ; 41$ (2011), p. 331 et 43 (2013), p. 300. 
2. L'architecture religieuse rurale en Languedoc Méditerranéen entre le IX siècle et le début du $\mathrm{XI}^{e}$ siècle (diocèses d'Agde, Béziers, Lodève, Maguelone et Narbonne), thèse d'histoire de l'art, sous la direction de G. Mallet, université Paul-Valéry Montpellier 3, soutenue en novembre 2016.

3. Le dossier a été suivi et subventionné par le SRA Occitanie et la Communauté de communes du Larzac-Lodévois. Voir É. ASTRUC, Le site de San Peyre: l'église (Saint-Maurice-Navacelles, 34), rapport final d'opération de sondages, SRA Languedoc-Roussillon, Montpellier, 2014.

4. Ordonné prêtre en 1952, Gérard Alzieu (1927-2009) fut le curé de nombreuses paroisses héraultaises, dont celle de Saint-Guilhem-le-Désert de 1972 à 2002. Passionné d'histoire et d'art roman, il a écrit de nombreux articles. Il fut en outre archiviste du diocèse de Montpellier de 1984 à 2008.

5. G. ALZIEU, Les églises de l'ancien diocèse de Lodève au Moyen Âge, Montpellier, 1998.

6. G. MILHAU, Navacelles, Montpellier, 1998.

7. L'église Saint-Vincent, située également sur la commune de Saint-Maurice-Navacelles, conserve deux linteaux monolithes en tuf creusé en arc plein cintre toujours en place. Dans le diocèse de Béziers, l'église Sainte-Marie-Madeleine, sise sur la commune de Vieussan, possède encore un linteau de même type.

8. La quarantaine de fragments mis au jour, représentant une dizaine d'objets, a été étudiée par Guergana Guionova, ingénieure d'études au LA3M-CNRS.

9. L'étude numismatique a été effectuée par Richard Pellé, ingénieur à l'Inrap.

\section{INDEX}

Mots-clés : sondages archéologiques, chapelle préromane

Index géographique : Languedoc méditerranéen, diocèse de Lodève

\section{AUTEUR}

\section{ÉLISABETH ASTRUC}

Docteur en histoire de l'art médiéval, rattachée au Centre d'études médiévales de Montpellier (EA 4583) 